Supporting Information for

\title{
Partition Analysis for Density-Functional Tight-Binding
}

\author{
Dmitri G. Fedorov \\ Research Center for Computational Design of Advanced Functional Materials \\ (CD-FMat), National Institute of Advanced Industrial Science and Technology (AIST),
}

Central 2, Umezono 1-1-1, Tsukuba, 305-8568, Japan. 
For covalent fragment boundaries, HOP was used in FMO. For fragmentations at $\mathrm{C}$ atoms (in $\mathrm{C} \alpha-\mathrm{C}$ and $\mathrm{C}-\mathrm{N}$ ), the standard set of hybrid orbitals was used, prepared for $\mathrm{CH}_{4}$. For S-S bonds, a new set was generated for $\mathrm{SH}_{2}$. The Pipek-Mezey localization scheme was used.

There are two 1-body PCM methods for FMO, PCM[1] and $\mathrm{PCM}<1>$. They differ in the way the charge transfer coupling is accounted for solvent in the total energy, but the induced solvent charges are the same. These two methods give the same atomic and solvent charges, and all PA components are the same (but FMO energies are different for $\mathrm{PCM}[1]$ and $\mathrm{PCM}<1>$ ).

Some idea of the cost of CT and PA calculations can be gained from the timings shown in Table S1. The CT state adds a very little cost over PL (16-17 s). PA requires solving PCM equations for each segment; the cost of PA for FMO2 is very substantial, but for FMO3 it is less than the FMO3 calculation. However, overall, calculations are very affordable, taking less than 20 minutes.

FMO and PA are efficiently parallelized so that their timings can be reduced when more than 1 core is used. For example, using 12 cores, PA/FMO3 calculation was done in $59 \mathrm{~s}$ (vs $415 \mathrm{~s}$ on 1 core, a speed-up of 7). 
Table S1. Timings (s) for the partition analysis ( 1 core of $2.8 \mathrm{GHz}$ Xeon CPU), crambin.

\begin{tabular}{|l|l|l|l|l|l|}
\hline method & timing & method & timing & method & timing \\
\hline FMO2/PL $^{\mathrm{a}}$ & 109 & FMO2/CT $^{\mathrm{a}}$ & 126 & PA/FMO2 & 413 \\
\hline FMO3/PL $^{\mathrm{a}}$ & 522 & FMO3/CT $^{\mathrm{a}}$ & 538 & PA/FMO3 & 415 \\
\hline full & 829 & & & PA/full & \\
\hline
\end{tabular}

${ }^{\text {a } U s i n g ~ t h e ~ l o c a l ~ s c r e e n i n g ~ m o d e l . ~}$

${ }^{\mathrm{b}}$ Additional cost of PA.

c Unfragmented. 